\title{
Correction to: Decreased urinary excretion of the ectodomain form of megalin (A-megalin) in children with OCRL gene mutations
}

\author{
Chikushi Suruda ${ }^{1}$. Shoji Tsuji ${ }^{1}$. Sohsaku Yamanouchi ${ }^{1} \cdot$ Takahisa Kimata $^{1} \cdot$ Nguyen Thanh Huan $^{2}$. \\ Hiroyuki Kurosawa $^{3} \cdot$ Yoshiaki Hirayama $^{3} \cdot$ Hiroyasu Tsukaguchi ${ }^{2} \cdot$ Akihiko Saito $^{4} \cdot$ Kazunari Kaneko $^{1}$
}

Published online: 18 January 2022

(c) The Author(s), under exclusive licence to International Pediatric Nephrology Association 2022

\section{Correction to: Pediatr Nephrol (2017) 32:621-625 \\ https://doi.org/10.1007/s00467-016-3535-x}

The original version of the above-mentioned article, unfortunately, has been recently found to contain a mistake. Throughout the article, all the values corrected by urinary creatinine in megalin including $\mathrm{C}$-megalin and A-megalin and $\beta 2$-microglobulin (BMG) were 10 times higher than the correct values and hundredth part of the correct values, respectively. The corrected Table 1 is presented below.

The original article can be found online at https://doi.org/10.1007/ s00467-016-3535-x.

Kazunari Kaneko

kanekok@hirakata.kmu.ac.jp

1 Department of Pediatrics, Kansai Medical University, 2-5-1

Shin-machi, Hirakata, Osaka 573-1010, Japan

2 Division of Nephrology, 2Nd Department of Internal Medicine, Kansai Medical University, Osaka, Japan

3 Diagnostics Research Department, Denka Innovation Center, Life Innovation Research Institute, Denka Co. Ltd, Tokyo, Japan

4 Department of Applied Molecular Medicine, Niigata University Graduate School of Medical and Dental Sciences, Niigata, Japan 
Table 1 Characteristics and results of the study subjects

\begin{tabular}{|c|c|c|c|c|c|}
\hline \multirow[t]{2}{*}{ Clinical and laboratory characteristics } & \multicolumn{5}{|c|}{ Case number/ patient age } \\
\hline & Case $1 / 5$ years & Case $2 / 8$ years & Case $3 / 9$ years & Case $4 / 12$ years & Case $5 / 15$ years \\
\hline Clinical diagnoses & LS & $\mathrm{LS}$ & $\mathrm{T} 2 \mathrm{D}$ & $\mathrm{T} 2 \mathrm{D}$ & LS \\
\hline \multicolumn{6}{|l|}{ Clinical features } \\
\hline Short stature & $-2.0 \mathrm{SD}$ & $-0.3 \mathrm{SD}$ & $-2.3 \mathrm{SD}$ & $-1.4 \mathrm{SD}$ & $-3.5 \mathrm{SD}$ \\
\hline Ocular abnormalities & - & cataract & - & - & cataract \\
\hline Mental retardation & + & ++ & - & - & + \\
\hline \multirow[t]{2}{*}{$O C R L$ mutation } & c. $1842 \mathrm{G}>\mathrm{A}$ & c. $2396 \mathrm{C}>\mathrm{T}$ & c. $278 \mathrm{~A}>\mathrm{G}$ & c. $1576 \mathrm{C}>\mathrm{T}$ & c. $208 \mathrm{G}>\mathrm{T}$ \\
\hline & $\begin{array}{l}\text { p.Trp641stop } \\
\text { exon } 17\end{array}$ & $\begin{array}{l}\text { p.Pro782Leu } \\
\text { exon } 21\end{array}$ & $\begin{array}{l}\text { p.Glu93Gly } \\
\text { exon } 5\end{array}$ & $\begin{array}{l}\text { p.Pro509Ser } \\
\text { exon } 15\end{array}$ & $\begin{array}{l}\text { p.Glu70stop } \\
\text { exon } 4\end{array}$ \\
\hline A-megalin (pmol/g Cr) $[77.76-479.2]^{\mathrm{a}}$ & 285.0 & 18.80 & 14.19 & 67.28 & 18.39 \\
\hline C- megalin (pmol/g Cr) $[0-3.31]^{\mathrm{a}}$ & 0.851 & 0.538 & 0.148 & 0.019 & 0.542 \\
\hline $\mathrm{BMG}(\mathrm{ng} / \mathrm{mg} \mathrm{Cr})[\text { normal }<219.2]^{\mathrm{a}}$ & $75.0 \times 10^{3}$ & $182.7 \times 10^{3}$ & $167.1 \times 10^{3}$ & $152.8 \times 10^{3}$ & $83.72 \times 10^{3}$ \\
\hline
\end{tabular}

OCRL: Gene encoding oculocerebrorenal syndrome of Lowe, A-megalin: urinary excretion of A-megalin corrected by creatinine, C-megalin: urinary excretion of $\mathrm{C}$-megalin corrected by creatinine, BMG: urinary excretion of $\beta 2$-microglobulin corrected by creatinine

LS: Lowe syndrome, SD: standard deviation, T2D: type 2 Dent disease

${ }^{\text {a }}$ The range of the 2.5th-97.5th percentile values for control children are presented in square brackets, except for the values for BMG, which are cited from Christensen et al. [10]

Publisher's Note Springer Nature remains neutral with regard to jurisdictional claims in published maps and institutional affiliations. 